\title{
A scuola di democrazia: insegnare storia contemporanea alla primaria
}

\author{
di Elena Mastretta
}

Set 18, 2019 | Didattica in classe, In evidenza $\mid \underline{0 \mid}$

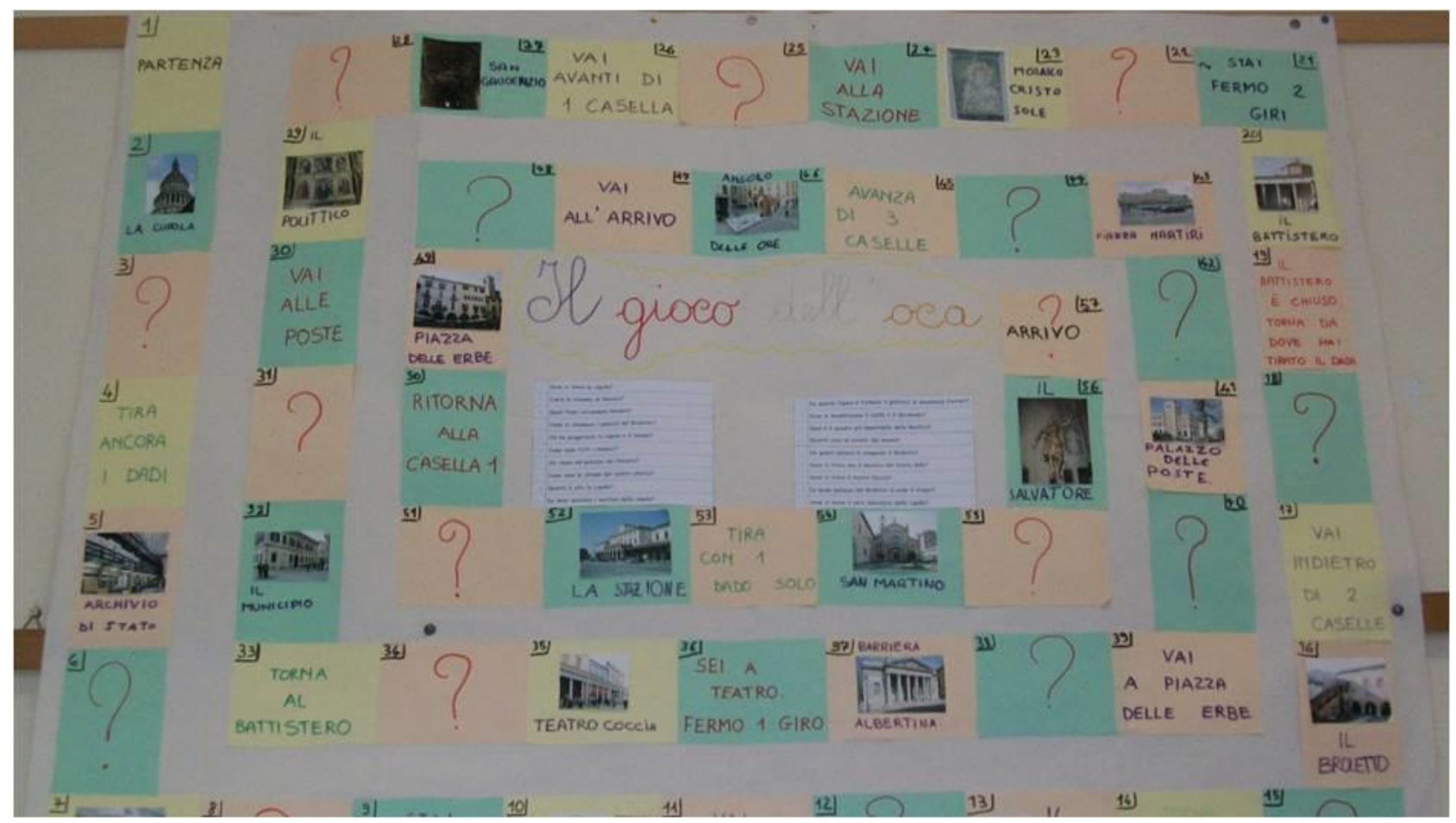

Heuberg 17 luglio 1944, Benito Mussolini passa in rassegna i reparti della divisione "Italia". Fotografia d'epoca, Pubblico dominio, $\underline{\text { Collegamento }}$

\begin{abstract}
L'articolo riporta l'esperienza pluriennale dei percorsi di storia contemporanea di classi della primaria organizzati nel novarese e nel Verbano Cusio Ossola attraverso visite guidate, incontri con testimoni e laboratorio di analisi delle fonti.

Insegnare storia contemporanea alla scuola primaria: un percorso nato da una richiesta

I "programmi scolastici" rendono talvolta problematico approfondire alla scuola primaria alcuni argomenti di storia contemporanea, che sono comunque ricompresi nell'insegnamento di Cittadinanza e Costituzione. La sperimentazione didattica avviata e qui illustrata parte da una specifica richiesta[1] per una classe quinta della primaria e, in accordo con tutti i soggetti coinvolti, ha previsto la stesura di un percorso lungo, con scadenze regolari, per permettere ai bambini di inserire in una cornice storica quanto appreso durante le visite guidate e i percorsi di approfondimento sulla storia della città svolti con le operatrici del Nucleo di Didattica Ambientale[2] già negli anni precedenti.
\end{abstract}




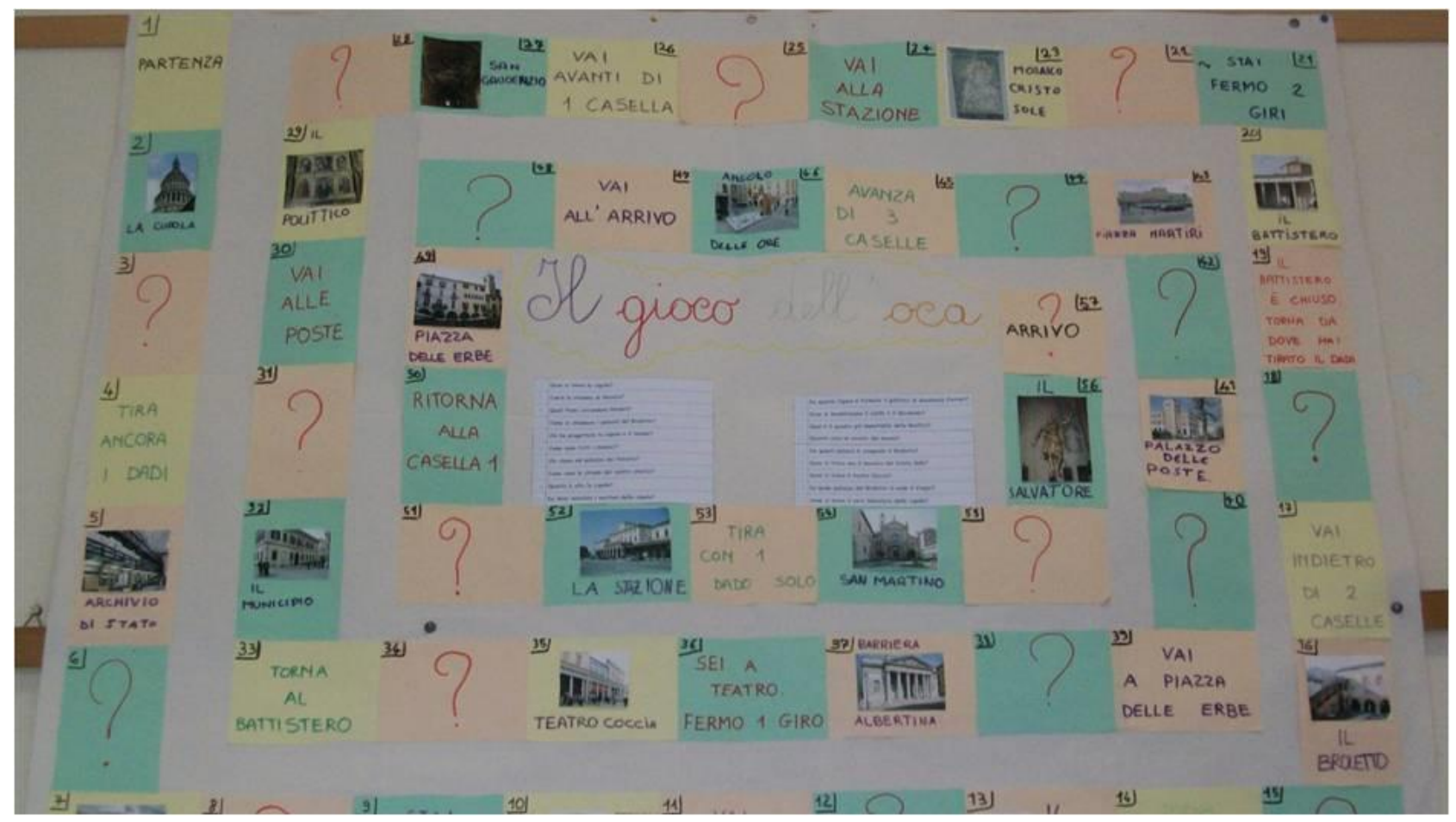

Ciò serviva anche per arrivare all'incontro con un testimone, alla preparazione di un breve discorso pubblico per il 25 aprile e alla stesura di una "Costituzione di classe"[3]. Già nel primo progetto, l'intenzione dell'insegnante era quella di avvicinare i bambini alle istituzioni con la partecipazione ad una cerimonia pubblica, cosa che in effetti avvenne, ma che solo nel 2017 si è configurata come un momento programmato nelle celebrazioni organizzate dal comune di Novara, che ha inserito l'intervento dei bambini nel programma ufficiale riportandolo negli inviti e nelle locandine.

Dopo la prima realizzazione del 2012, il percorso ha continuato ad essere richiesto dalle scuole primarie e secondarie di primo grado in una sorta di "versione ridotta", fatta di cinque incontri modulabili a scelta dell'insegnante, a cura del Nucleo di Didattica Ambientale e della sezione didattica dell'Istituto Storico Piero Fornara andando a configurarsi come proposta didattica ripetibile[4].

\section{La scansione del percorso}

Dopo una serie di riunioni tra i diversi soggetti formatori, l'ossatura del percorso risultò questa:

- Il Novecento: un secolo diverso dai precedenti.

- I regimi totalitari: cosa sono, la vita sotto la dittatura.

- Preparazione all'incontro con il testimone.

- Visita guidata a Novara: i luoghi del fascismo.

- Incontro testimone: Il Comandante BELLINI[5]

- Visita guidata a Novara: i luoghi della Resistenza.

- Figure novaresi della resistenza (Marcella Balconi, Giulio Biglieri, Monsignor Ossola, Rina Musso, Piero Fornara, tra gli altri).

- Indagine toponomastica e sui monumenti presenti in città, con ripresa del "gioco dell'oca" per la conoscenza della città realizzato dalla classe due anni prima.

- Visita a Casa Fornara (sede dell'Istituto Storico Piero Fornara).

- Vita quotidiana durante la guerra: la scuola, il cibo, il tempo libero. 
- Come è nata la Costituzione e preparazione al 25 aprile.

- Partecipazione cerimonia pubblica in Broletto il 25 aprile.

- Riflessione collettiva sul 25 aprile.

- La Novara del dopoguerra: la libertà ritrovata e la presenza del Centro Raccolta Profughi della Caserma Perrone.

- Lavoro in classe: scriviamo la nostra costituzione (due incontri).

- Visita a luoghi della memoria significativi fuori del tessuto cittadino: Fondotoce[6]

Ma quante ore ci vogliono?

Grazie alla capacità organizzativa dell'insegnante, che ha costruito intorno a questo percorso buona parte delle altre attività curricolari, è stato possibile prevedere incontri a cadenza settimanale. Nei mesi di svolgimento del progetto i contenuti storici che venivano affrontati, ad esempio, erano poi oggetto delle tipologie testuali che era previsto affrontare[7], così come su questi argomenti si sono concentrate le produzioni artistiche. A titolo esemplificativo, proponiamo alcune delle poesie scritte dagli alunni della classe VA della scuola di Bollini che ha svolto il percorso nella sua seconda realizzazione, poesie che sono state lette in occasione della Cerimonia per il 25 aprile 2017 a Novara. Alunne e alunni hanno steso un testo facendo riferimento a quanto era stato presentato dagli esperti sulla Liberazione di Novara e dopo avere visitato i luoghi della Resistenza in città.

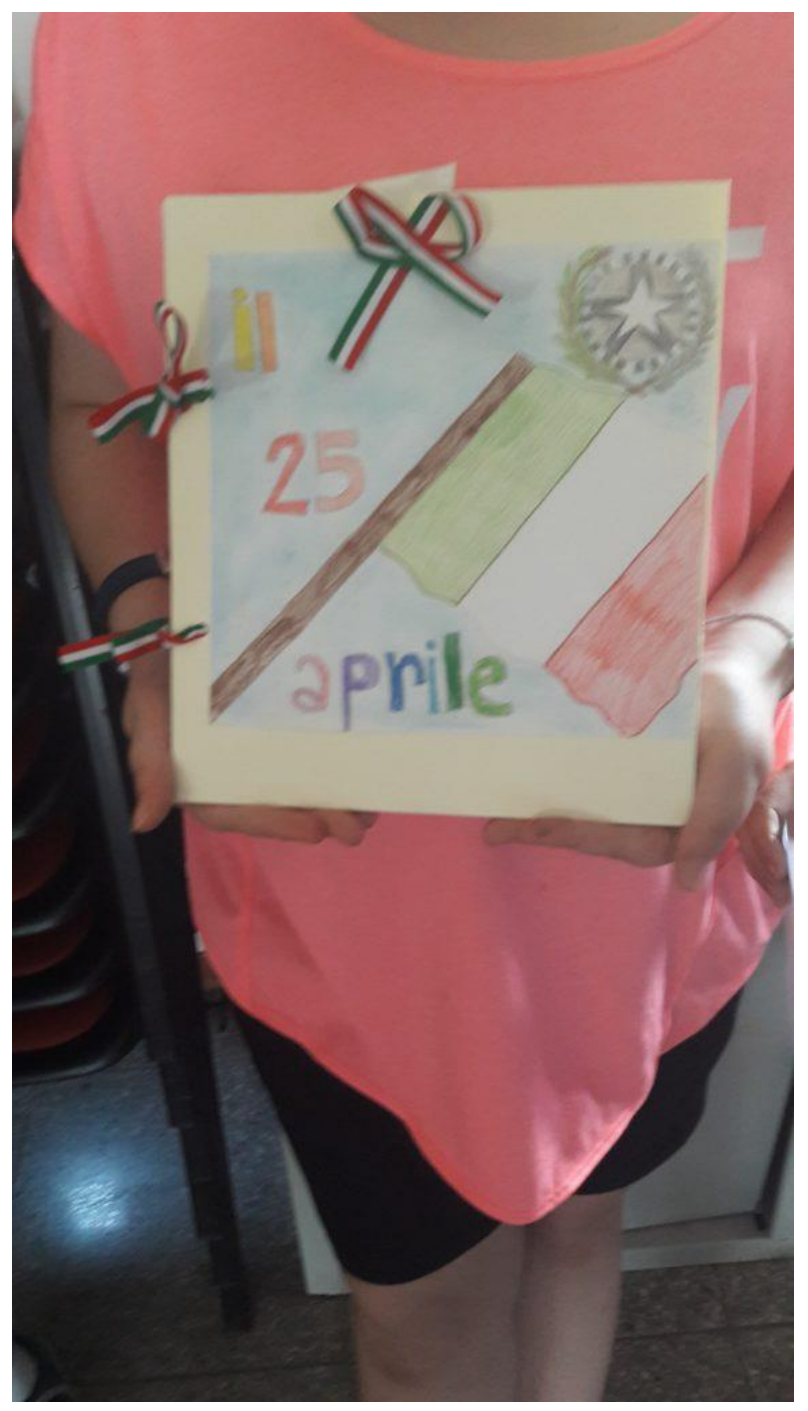




\section{Sogno di un partigiano}

Io partigiano,

sto combattendo per una "cosa" importante.

Non è un giocattolo, una bambola, una palla, no non lo è.

E' una cosa speciale che tutti dovrebbero avere... la libertà.

Spero che un giorno

i miei discendenti possano capire

quanta fatica ho fatto per avere questa "cosa".

Ieri ero un bambino,

oggi ho 16 anni ma continuerò a combattere

finché non realizzerò il mio sogno.

Tessa Peoples

\section{Che gioia la Liberazione}

Il 25 aprile 1945 ,

sapevo bene che i partigiani ce l'avrebbero fatta,

ci speravo, ci credevo,

e non ero il solo.

Un'intera città era lì ad acclamarli, ad incoraggiarli.

Speravo con tutto il cuore nella liberazione, e così è stato,

ancor meglio di come credevo.

Niente uccisioni, niente spari, nessun bagno di sangue, solo parole e discussioni che hanno mandato via i Tedeschi.

Dopo la liberazione, nelle strade, c'erano amore felicità e gioia.

Niente tristezza o ansia e paura.

Nessuno si è vendicato, si è solo fatta giustizia

in una situazione che ne aveva bisogno.

Diego Gila

$$
\text { E' cambiato qualcosa }
$$

Oggi ho sentito qualcosa di diverso, ieri mi proteggevo dall'arma della morte oggi provo emozioni diverse, mi sento vittorioso, 
ieri ero nelle ali della paura,

oggi sento speranza nella vita, ieri sentivo ansia,

oggi vedo gente che applaude,

ieri vedevo persone che strappavano vite,

oggi provo amore per tutti,

ieri mi ero quasi dimenticato di cosa fosse l'amore,

oggi ho fretta di entrare in città

perché è il 25 aprile.

Lucia Ratti

Le poesie sono state raccolte dall'insegnante in un volumetto che è stato poi presentato ai genitori in occasione dell'esposizione del lavoro fatta a loro beneficio a giugno 2017.

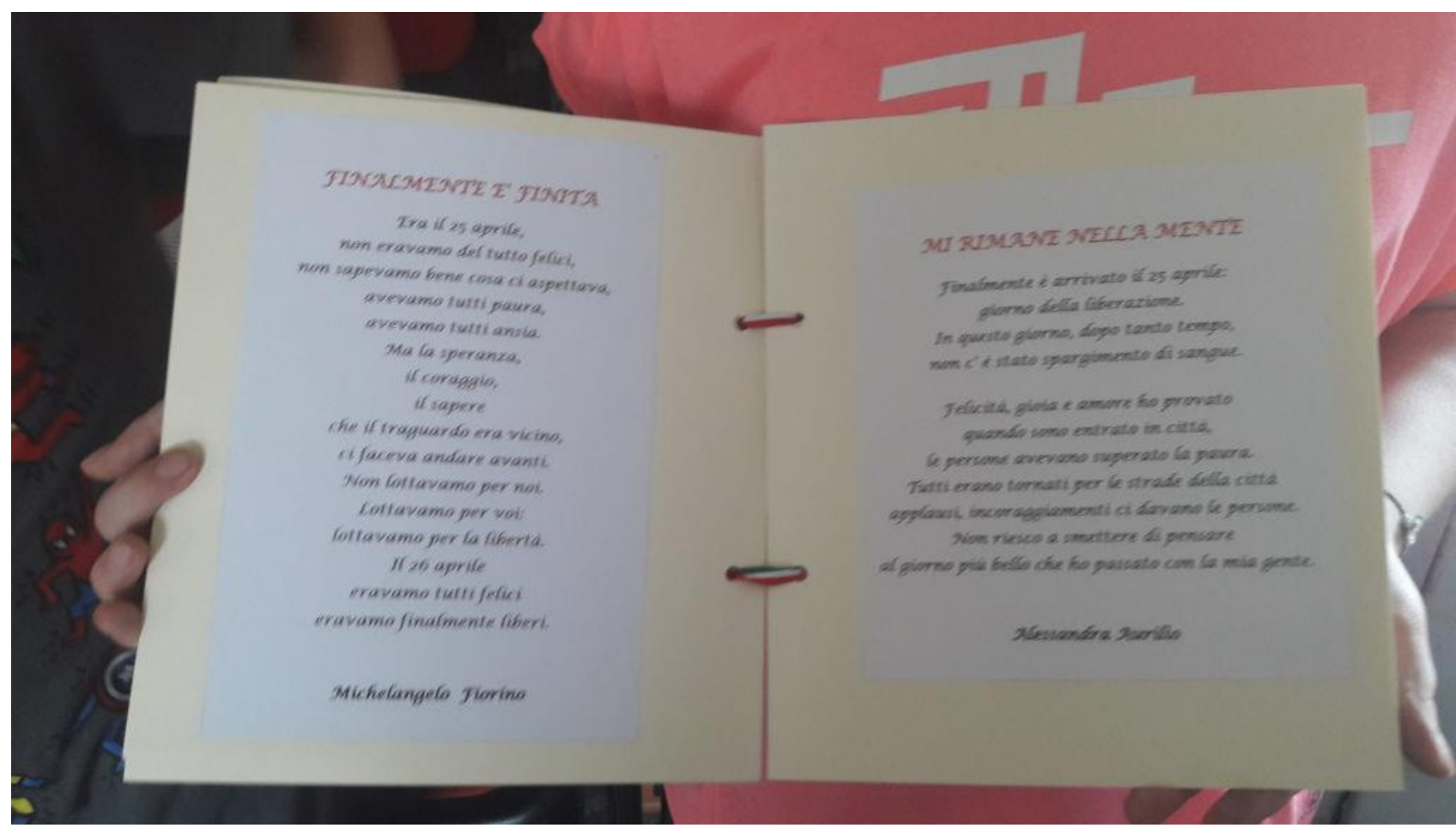

La loro stesura da parte dei ragazzi dimostra l'interdisciplinarietà che può crearsi nella realizzazione di questo tipo di percorsi.

Il numero di ore dedicate al progetto, nell'a.s 2011/12 una trentina solo in presenza degli esperti esterni, ha rappresentato una criticità nella riproposizione. La scelta dell'insegnante Crescimbeni nelle due realizzazioni del progetto è stata quella di non pensare al percorso di approfondimento di storia contemporanea come ad un argomento estraneo all'attività didattica. Scelta che si è rivelata possibile e che la nostra esperienza indica riproponibile anche in altri contesti. Soffermarsi su questi argomenti significa lavorare con i bambini su temi che sono trasversali ai diversi insegnamenti disciplinari, inserendoli in una contestualizzazione storica che ha lo scopo di rendere più chiaro e consapevole il percorso per la classe. 
Le modalità di svolgimento degli incontri, in genere organizzati nel pomeriggio, per due ore consecutive, sono state improntate alla didattica informale e all'interattività. Le figure che hanno condotto i diversi interventi hanno mantenuto stretti contatti tra loro e concordato tutti i contenuti da proporre ai bambini, cercando di evitare sovrapposizioni, ma utilizzando la ripresa di argomenti trattati da altri in diverso modo o in differenti contesti come domanda-stimolo iniziale per le diverse tappe del percorso. In tutti i casi in cui è stato possibile, chi non conduceva direttamente l'incontro era comunque presente e verbalizzava ciò che accadeva[8]. Spesso l'incontro si concludeva con la realizzazione di un cartellone, che veniva poi appeso in classe e descritto dai bambini a chi interveniva nel successivo appuntamento.
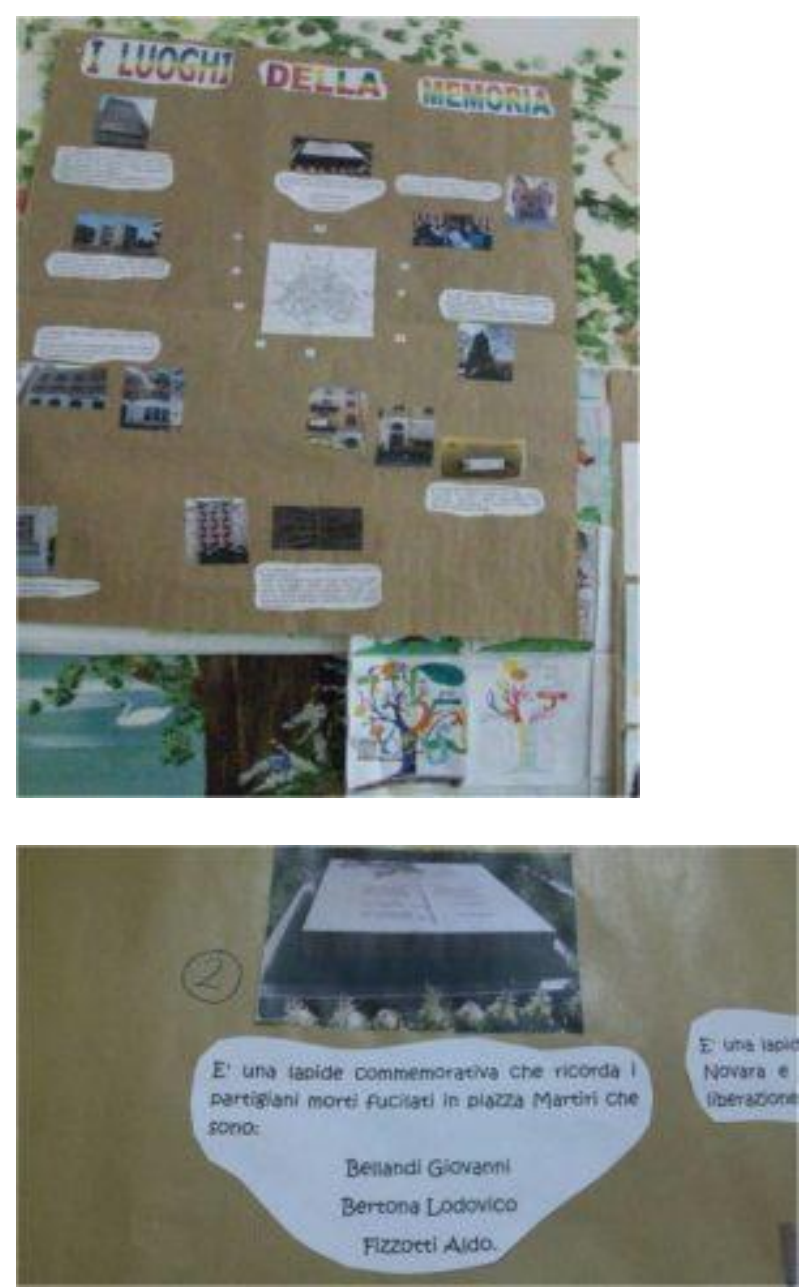

Gli studenti hanno incontrato figure abituate al dialogo con i più piccoli, ma anche studiosi, testimoni. In tutti questi casi si è avuta l'accortezza di spiegare i termini specialistici che venivano utilizzati e di accompagnare l'intervento, che avrebbe potuto risultare teorico, noioso o difficile, con immagini, esempi, oggetti. 
Le scuole di Novara, come accennato, hanno la possibilità già dal terzo anno della primaria di fare percorsi di conoscenza della città con il Nucleo di Didattica Ambientale. Questa esperienza pregressa facilita lo svolgimento di moduli di storia del Novecento. In tutti gli altri casi, abbiamo constatato che è molto utile partire da qualcosa di concreto: l'intitolazione della scuola o di una via ad essa vicina, la presenza, nelle immediate vicinanze, di un monumento, di un edificio che possano essere la partenza per una narrazione storica. Di norma, almeno una di queste condizioni si verifica e il primo incontro parte con la domanda "Chi era il tale personaggio?" piuttosto che "Voi sapete qual era l'utilizzo di questo edificio?’[9]

Si prosegue poi con un numero minimo di cinque incontri condotti dalla sezione didattica dell'Istituto e/o da altri esperti: "Il Novecento: la lunga storia del secolo breve"; "Novara 19251945"; "Luoghi e personaggi della Resistenza novarese"; "L'Assemblea costituente e i 12 principi fondamentali"; "Scriviamo la costituzione di classe". Con questa formula si lasciano all'insegnante di classe delle schede e delle indicazioni di lavoro per condurre delle discussioni su quanto ascoltato e per realizzare delle produzioni da parte dei ragazzi. Qualora la classe svolga più di 5 incontri con gli esperti, la rielaborazione viene condotta insieme.

Che materiali usare?

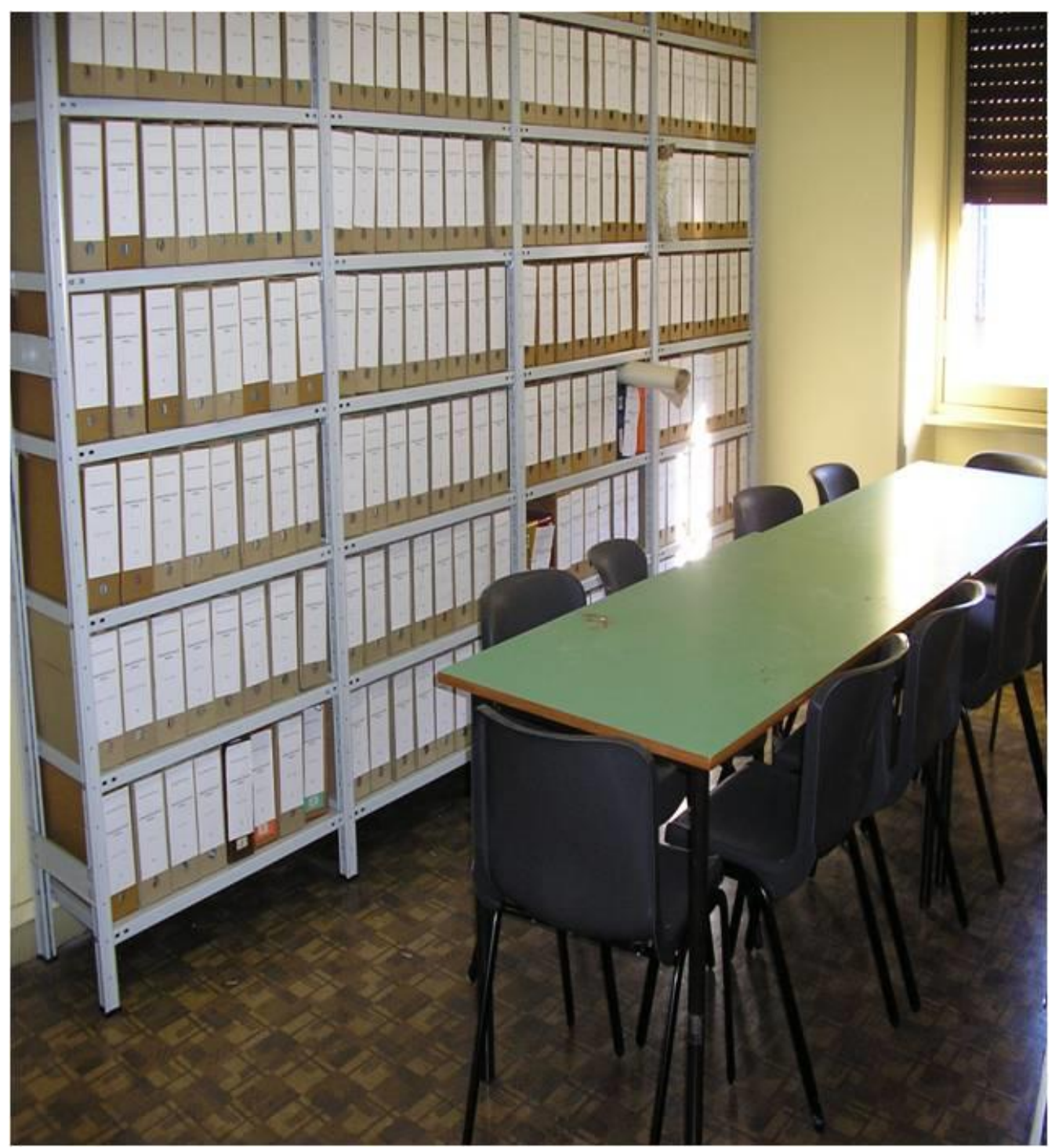


L'esperienza fatta dimostra che è possibile usare anche con i bambini materiali d'archivio e pubblicazioni che spesso gli Istituti Storici curano su questi temi, adattandole al linguaggio e alle conoscenze dei bambini, come I Fili della Memoria e Profughi, nati per un pubblico adulto[10], avendo l'accortezza di rispondere mano che emergono a tutti i dubbi dei bambini modificando il percorso a seconda delle loro esigenze. Importante è stato fare tesoro dell'incontro con il testimone, che nel percorso del 2012 è stato filmato e caricato su youtube per favorirne la massima diffusione e successive possibilità di utilizzo anche da parte di altre classi[11].

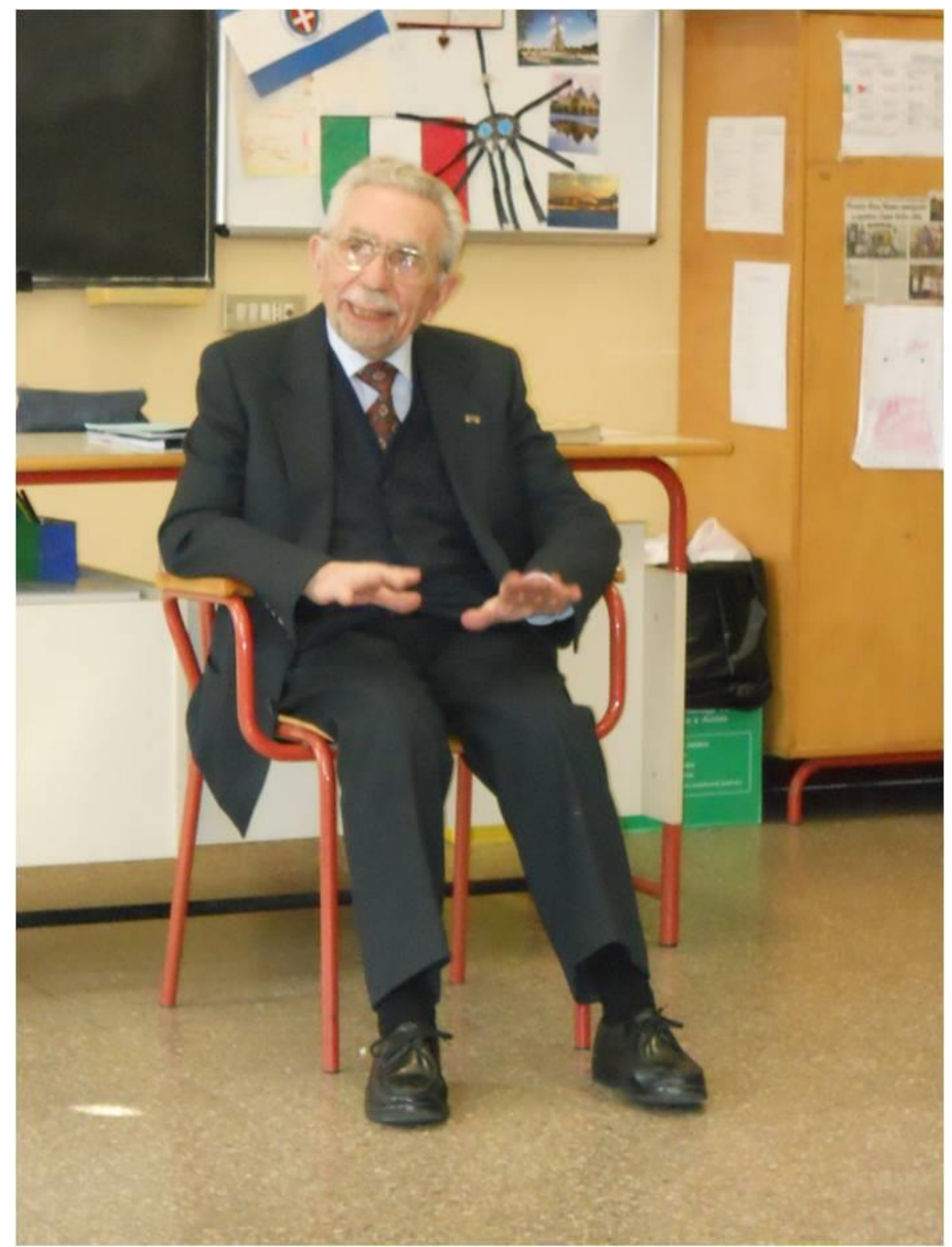




\section{Gli oggetti}

In alcuni casi, si è fatto ricorso a degli oggetti che potessero aiutare i bambini a comprendere meglio il periodo di cui si stava parlando: non solo fotografie o lettere selezionati dagli archivi dell'Istituto Storico, ma oggetti che potessero essere toccati (e interrogati). Per parlare del pericolo dei bombardamenti e dei rifugi antiaerei, ad esempio, si è portata in classe una valigetta "didattica" con modelli di bombe che veniva usata nel dopoguerra per sensibilizzare i più piccoli alla possibilità di trovare, giocando, una bomba inesplosa.

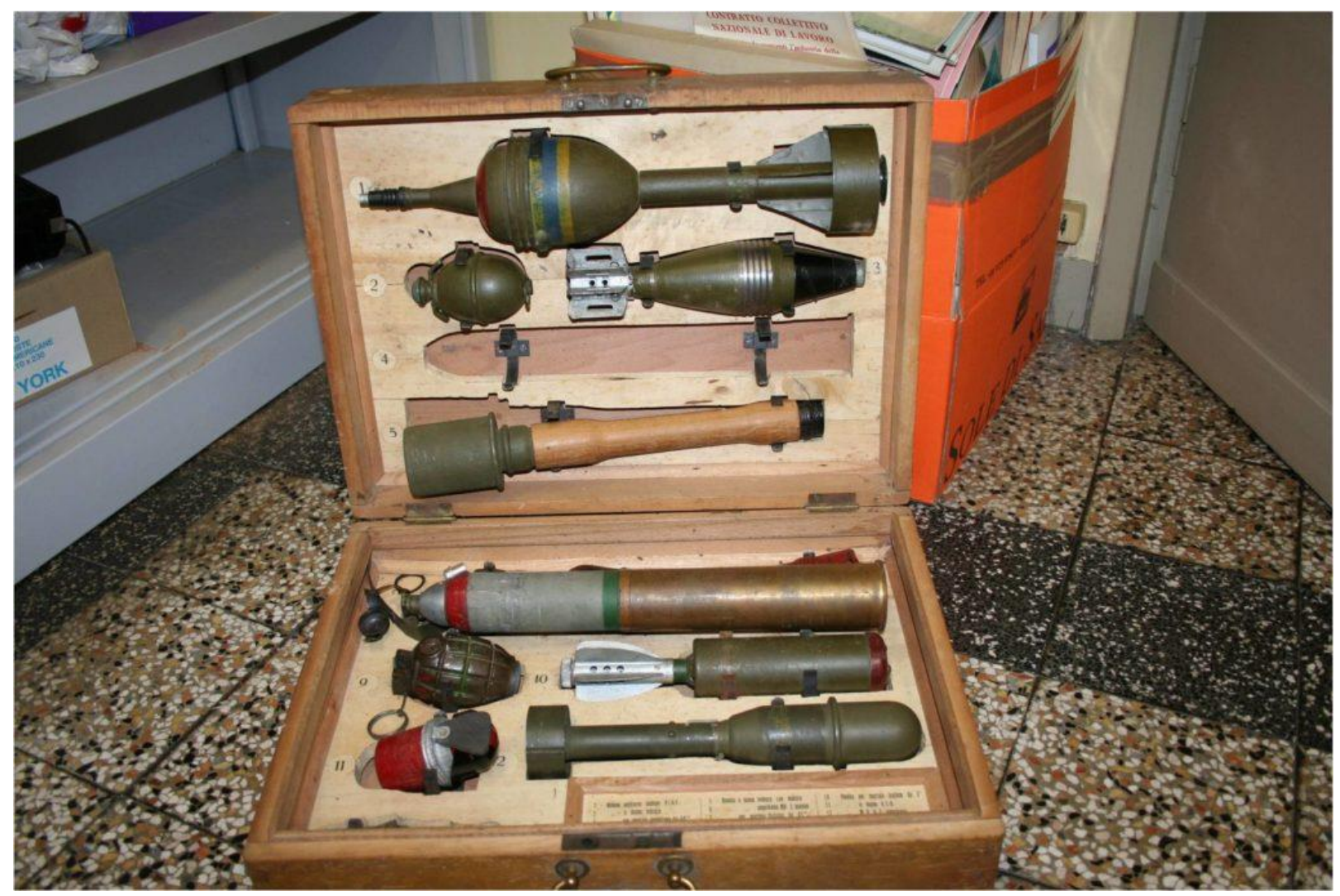

Si tratta di un corredo che alcune scuole possiedono ancora e che ha suscitato grande interesse, permettendo anche di fare qualche riflessione sui bombardamenti che si verificano oggi e di cui parlano i telegiornali. Quando abbiamo affrontato questo argomento, abbiamo mostrato ai bambini una foto del bombardamento di Novara. 


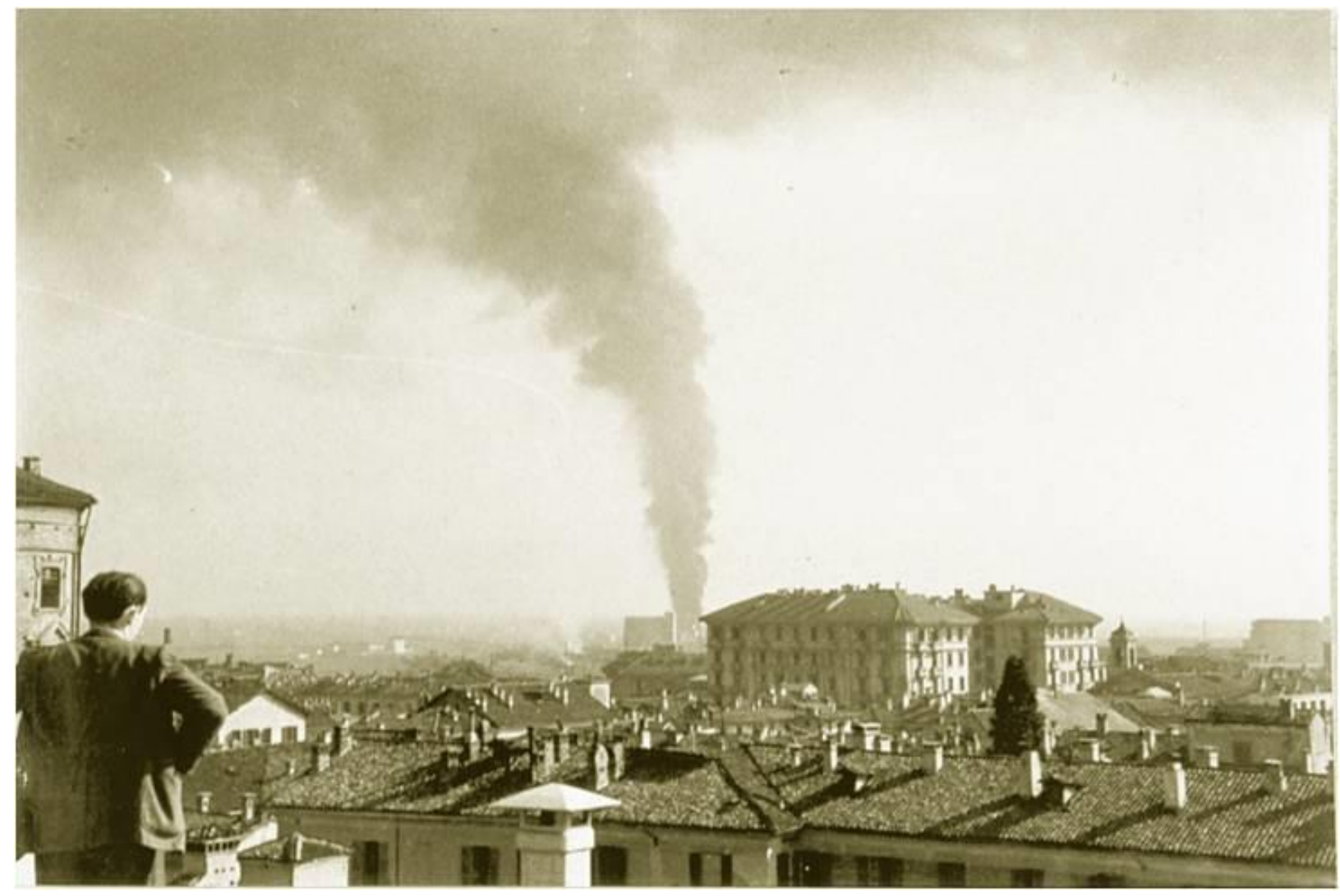

Facendo la lettura dell'immagine e abbiamo usato come spunto iniziale la sosta davanti alla "R" del rifugio antiaereo di via Dominioni che fa parte dei percorsi guidati in città.

Allo stesso modo, per parlare della scuola in epoca fascista si sono fatti sfogliare ai bambini i testi dell'epoca: il confronto con i loro libri li ha subito portati a comprendere il significato di termini come "propaganda" e "censura".

\section{Uso delle fonti: le foto}

Le foto sono state tra i materiali d'archivio più utilizzati in questo percorso, soprattutto quelle del Fondo Bonzanini[12].

I bambini della scuola primaria conoscono i diversi tipi di fonti (materiali, documentarie, iconografiche, orali). Si mostrano loro un certo numero di fotografie precedentemente scelte e li si invita a indicare il luogo in cui l'immagine è stata scattata (per favorire il riconoscimento del luogo si è predisposta una serie di immagini scattate dalla stessa prospettiva ai giorni nostri). Una volta che il luogo è stato individuato, si confrontano l'immagine storica e quella attuale per percepirne somiglianze e differenze. L'esperto passa poi a raccontare la storia dei personaggi che appaiono nella fotografia, utilizzando in tutti i casi in cui è possibile la "voce del personaggio" con stralci da suoi scritti o carteggi, oppure ricorrendo alla descrizione che di lui fanno le fonti dell'epoca, soprattutto i giornali[13]. 
Alla fine di questo lavoro, l'immagine viene collocata su di un cartellone o nel quaderno dei bambini, a seconda del tipo di documentazione scelto dall'insegnante, con il riferimento di autore, luogo, personaggio ritratto, data dello scatto.

Spesso, al personaggio sono state fatte delle intitolazioni in città e a questo punto "lo si va a cercare". Ad esempio, una tappa dei percorsi in città è davanti alla statua di Monsignor Leone Ossola. Parlando di guerra e resistenza a Novara la figura carismatica del Vescovo di Novara viene sempre descritta e il ritratto scultoreo si presta ad essere confrontato con le numerose fotografie che ritraggono Ossola nei giorni immediatamente successivi al 26 aprile 1945 impegnato nelle trattative di resa[14]. Spesso il lavoro di restituzione in classe prevede di far realizzare agli studenti a loro volta un ritratto di Monsignor Ossola, inserendo nel percorso anche la disciplina artistica.
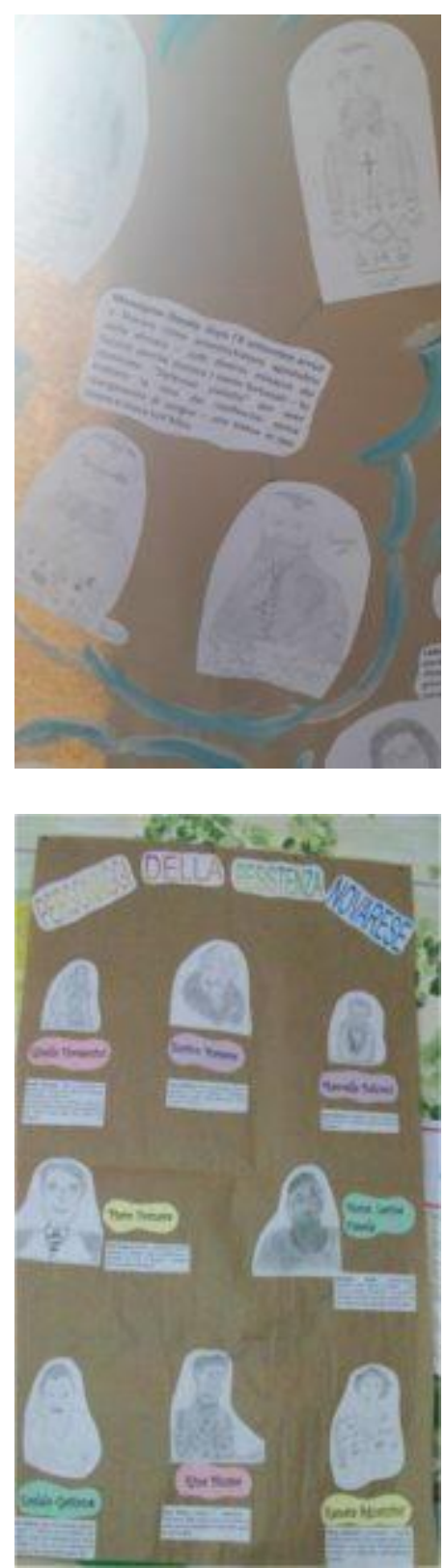
L'aver ripetuto il percorso nella sua interezza a cinque anni di distanza ha permesso di operare in classe con maggiore sicurezza, avendo nel tempo predisposto materiali e sviluppato strategie di intervento che coprono una casistica ampia delle difficoltà che si possono incontrare quando si parla a ragazzi piccoli di totalitarismi, guerra. Non è tanto, come qualcuno sostiene, la presenza di bambini stranieri nelle classi a rendere "difficile" la trattazione di questi argomenti, piuttosto le false credenze che ancora circolano su di essi e che molto spesso hanno attecchito nelle famiglie dei bambini, che potrebbero quindi doversi confrontare con due diverse letture dei fenomeni presi in esame: quella scolastica e quella familiare. Riteniamo pertanto utile che la realizzazione del percorso sia condivisa con le famiglie, con la specificazione che non verranno forniti "giudizi", ma chiavi di lettura nell'ottica della cittadinanza attiva. A tale scopo i percorsi vengono annunciati e approvati nella programmazione e negli incontri periodici con i genitori ed è utile concluderli con una restituzione pubblica all'interno di momenti istituzionali o appositamente creati[15].

[inserire foto presentazione genitori 2 e foto Argante Bocchio]

Note:

[1] Nel 2012, su sollecitazione dell'insegnante Laura Crescimbeni, in servizio presso il plesso di via Bollini-Istituto comprensivo Duca d'Aosta-Novara, che svolgeva con la sua classe V primaria un approfondimento sulla città di Novara nel periodo storico 1940-45 con la sezione Artistica del Nucleo di Didattica Ambientale del Comune di Novara, è iniziata la costruzione di percorsi didattici sul ' 900 poi riproposti in collaborazione dai diversi soggetti che li hanno avviati. Nel periodo 2012/2017 sono stati coinvolti una cinquantina di gruppi classe delle scuole primarie e secondarie di primo grado di Novara città, e una ventina di classi del novarese e del Verbanio Cusio Ossola. Il Nucleo di Didattica ambientale opera solo nel territorio del Comune di Novara e negli altri ambiti territoriali il percorso è proposto in modo autonomo dalla sezione didattica dell'Istituto Storico Piero Fornara. Nell'a.s. 2016/17 la maestra Crescimbeni ha riproposto il progetto alla classe $\mathrm{V}$ partecipando in modo ufficiale con la classe alle Celebrazioni del 25 aprile che il Comune di Novara organizza presso il Broletto. Per l'occasione, i bambini hanno letto alcune poesie da loro scritte appositamente per la cerimonia.

[2] Attualmente il Nucleo, attivo da una trentina d'anni con una ricca proposta di iniziative per scuole e pubblico, impegna una ex insegnante comunale per la sezione Artistica e due per la sezione Ambientale, dipendenti dal settore istruzione del Comune di Novara. L'ultimo pensionamento risale alla primavera 2019, quindi dall'a.s. 2019/20 il servizio probabilmente muterà forma.

[3] La stesura della "Costituzione di classe", in particolare, si è poi sviluppata, nel corso degli anni successivi, come un percorso laboratoriale a sé stante proposto soprattutto alle classi di scuola secondaria di primo grado.

[4] Il percorso è poi stato realizzato da circa un centinaio di classi fino a giugno 2019, di cui un terzo della scuola primaria. 
[5] Vincenzo Grimaldi è nato a Caltagirone (CT) nel 1922, ma dal 1962 ha vissuto a Novara, dove ha prestato servizio nel Corpo delle Guardie di Pubblica Sicurezza con il grado di Maresciallo. In pensione dal 1977, ha fatto parte dell'Associazione Nazionale Partigiani d'Italia. Dal 1985 al 2000 ha svolto l'incarico di Amministratore del periodico mensile Resistenza Unita. Dopo l' 8 settembre 1943 è entrato a far parte della resistenza con il nome di battaglia Bellini ed è stato vice comandante della $181^{\circ}$ Brigata Garibaldi. Autore del volume Tutti pazzi o tutti eroi, Memorie del Comandante Bellini, 8 settembre 1943 - 25 aprile 1945, edito da Bonanno. Scomparso a dicembre 2016, il Comandante è stato ricordato dalla città in una giornata a lui dedicata il 21 maggio 2017, giorno in cui avrebbe compiuto 95 anni. Nel 2017 i bambini hanno incontrato Argante Bocchio, il partigiano Massimo.

[6] I numerosi interventi in classe e le visite guidate sono stati condotti dalle due operatrici del Nucleo di Didattica Ambientale del Comune di Novara, Anna Canetta e Patrizia Spagni, dalla docente in utilizzo presso la sezione didattica dell'Istituto, Elena Mastretta e dal Direttore Scientifico Giovanni Cerutti. L'incontro sulle figure Novaresi della Resistenza è stato condotto dal dottor Mauro Begozzi, che fino al luglio 2011 ha diretto l'Istituto Storico Piero Fornara.

[7] Dopo l'incontro con Vincenzo Bellini i ragazzi hanno realizzato un articolo di giornale, altro esempio di trasversalità del progetto.

[8] Tutto il percorso è stato documentato dall'insegnante di classe e dalla responsabile della sezione didattica dell'Istituto Storico con un diario di bordo e con delle fotografie ed ha poi costituito non solo la base per i successivi percorsi proposti in collaborazione tra Istituto Storico e NDA del Comune di Novara nelle classi, ma anche il nucleo di un corso di formazione per insegnanti realizzato nel 2013 dagli stessi soggetti ideatori del percorso ed è stato proposto, come esempio di buona pratica didattica, in diversi contesti formativi. Tra il 2013 e il 2014, oltre al corso di formazione realizzato presso la scuola Bollini di Novara, l'Istituto storico ha promosso due interventi di formazione rivolti ad insegnanti sul tema "insegnare Cittadinanza e Costituzione" a Grignasco in collaborazione con l'Istituto storico di Biella e Vercelli e a Borgomanero, in collaborazione con l'amministrazione locale, entrambi frequentati da insegnanti della primaria e della secondaria di primo grado.

[9] La scuola Bollini si trova vicino a via Biglieri e alla questura, un edificio di epoca fascista. In altre situazioni abbiamo svolto i percorsi in scuole intitolate ai partigiani Fratelli Di Dio, alle vittime dell'Olocausto del Lago Maggiore Fratelli Fernandez Diaz, a Pajetta, solo per fare alcuni esempi.

[10] In particolare abbiamo usato I Fili della Memoria e Profughi, nati per un pubblico adulto. I fili della memoria è una pubblicazione dell'Istituto Storico Piero Fornara curata da Antonella Braga nel 2001. Il testo, cui si aggiunge un DVD con i percorsi di visita, è esaurito, ma consultabile presso diverse biblioteche, tra cui quella dell'Istituto. Il DVD Profughi, anch'esso realizzato dall'Istituto Storico Piero Fornara a cura di Eligio Pastrovicchio e Antonio Leone, raccoglie testimonianze di persone che hanno vissuto al Centro Raccolta Profughi della Caserma Perrone di Novara ed è allo stesso modo fuori commercio. Si tratta di ricerche rivolte ad un pubblico adulto, ma ricche di spunti per il lavoro nelle scuole primarie.

[11] Vincenzo Grimaldi ha incontrato gli alunni della maestra Laura Crescimbeni il 15.3.2012. In quell'occasione la sezione didattica dell'Istituto Storico Piero Fornara chiese al regista Enrico Omodeo Salé di riprendere l'incontro. Da allora il video è caricato su yuotube (https://www.youtube.com/watch?v=t3xm-n6kajQ) e si è rivelato prezioso in tutte le occasioni in cui non è stato possibile un incontro diretto tra il testimone e gli studenti. Dopo la scomparsa del 
Comandante, è diventato per noi ancora più importante. Nell'a.s. 2016/17 La classe ha incontrato come testimone Argante Bocchio, nome di battaglia Massimo.

[12] Umberto Bonzanini ha realizzato una vera e propria campagna fotografica della Liberazione di Novara e le sue foto sono spesso utilizzate a scopo didattico dall'Istituto Storico Piero Fornara. Lo studio del fotografo, come oggi, si trovata in Corso Cavour 15, lo stesso palazzo in cui abitava il Professor Piero Fornara, uomo chiave della resistenza novarese, cui è dedicato l'Istituto storico delle province di Novara e del VCO. Fu proprio Fornara ad avvisare Bonzanini che il 26 aprile 1945 sarebbe successo qualcosa di memorabile in città, consigliandolo il giorno precedente di uscire di casa la mattina successiva con ben due apparecchi fotografici. Questo ha permesso una documentazione fotografica della Liberazione di Novara estremamente puntuale e molto interessante a scopi scientifici.

Il Fondo Bonzanini, da tempo depositato presso l'Istituto Storico Piero Fornara è stato di recente digitalizzato ed una scelta di immagini è stata pubblicata in volume a cura del figlio Eugenio. Si tratta di immagini molto note ai novaresi, in quanto spesso utilizzate come elemento iconografico dei manifesti delle celebrazioni ufficiali, che costituiscono un patrimonio di informazioni per tutti e che meritano una ancor maggiore diffusione e analisi.

[13] Nel nostro ambito territoriale di intervento fondamentale da questo punto di vista è il testo di Enrico Massara Antologia dell'antifascismo e della resistenza novarese.

[14] Diego Gila nella sua poesia scrive: "Niente tristezza o ansia e paura./Nessuno si è vendicato,/si è solo fatta giustizia/in una situazione/che ne aveva bisogno". I riferimenti alle trattative con tedeschi ed angloamericani di Monsignor Ossola e Piero Fornara che sono state descritte ai ragazzi negli incontri con gli storici sono qui chiarissimi.

[15] Per diversi anni il Comune di Novara ha organizzato per le scuole cittadine l'evento "scuole in piazza", nel quale ogni scuola e ogni classe aveva la possibilità di esporre i lavori realizzati durante l'anno. Lo stesso accade per i plessi ricompresi nell'IC del Vergante, che organizza annualmente una Festa d'Istituto allo stesso scopo. Nello specifico, molte delle classi che hanno partecipato a questo percorso hanno poi manifestato la volontà di diffonderlo con differenti modalità: partecipazione alle celebrazioni civili, spettacoli, realizzazione di libri d'autore e invito diretto ai genitori. Nell'a.s. 2015/16 si è organizzato un piccolo concorso per raccogliere questi materiali e farli entrare in un archivio di produzioni didattiche che vuole essere di supporto alle classi che seguono il percorso soprattutto nelle forme più brevi. http://www.isrn.it/concorsocostituzione-chepassione/.

A giugno 2017 Laura Crescimbeni durante un pomeriggio ha allestito l'esposizione dei lavori realizzati dai bambini nel percorso invitando le famiglie, gli esperti dell'Istituto Storico Piero Fornara, le docenti del Nucleo di Didattica Ambientale e Argante Bocchio, il testimone partigiano che i bambini avevano incontrato in questa edizione. Numerose le presenze delle famiglie, che hanno ricevuto la spiegazione del lavoro direttamente dai bambini, che si erano distribuiti in sezioni tematiche all'interno della stanza. 